\title{
BMJ Open Cost-effectiveness of custom-made insoles versus usual care in patients with plantar heel pain in primary care: cost-effectiveness analysis of a randomised controlled trial
}

Nadine Rasenberg (D , ${ }^{1}$ Marienke van Middelkoop, ${ }^{1}$ Sita M A Bierma-Zeinstra, ${ }^{1}$ Mohamed El Alili, ${ }^{2}$ Patrick Bindels, ${ }^{1} \mathrm{~J} \mathrm{Bosmans}^{2}$

To cite: Rasenberg N, van Middelkoop M, BiermaZeinstra SMA, et al. Costeffectiveness of custom-made insoles versus usual care in patients with plantar heel pain in primary care: cost-effectiveness analysis of a randomised controlled trial. BMJ Open 2021;11:e051866. doi:10.1136/ bmjopen-2021-051866

- Prepublication history and additional supplemental material for this paper are available online. To view these files, please visit the journal online (http://dx.doi.org/10.1136/ bmjopen-2021-051866)

Received 30 March 2021 Accepted 18 October 2021

A) Check for updates

(c) Author(s) (or their employer(s)) 2021. Re-use permitted under CC BY-NC. No commercial re-use. See rights and permissions. Published by BMJ.

${ }^{1}$ Department of General Practice, Erasmus Medical Center, Rotterdam, Zuid-Holland, The Netherlands

${ }^{2}$ Department of Health Sciences, Vrije Universiteit Amsterdam, Amsterdam, Noord-Holland, The Netherlands

Correspondence to

Nadine Rasenberg;

n.rasenberg@erasmusmc.nl

\section{ABSTRACT}

Objectives To evaluate the cost-effectiveness of custommade insoles compared with general practitioner (GP)-led usual care after 26 weeks of follow-up in individuals with plantar heel pain (PHP) from a societal perspective.

Design Cost-effectiveness analysis of a double-blinded randomised controlled trial.

Setting General practice in the Netherlands.

Participants 116 participants with PHP for at least 2 weeks, aged 18-65 years and presenting to the GP. Interventions Participants were randomised to GP-led usual care $(n=46)$ or referral to a podiatrist for treatment with a custom-made insole $(n=70)$. Participant randomised to a sham insole $(n=69)$ were excluded from this analysis.

Primary and secondary outcomes Outcomes comprised pain during rest and activity, and quality of life. Costs included healthcare and lost productivity costs. Statistical uncertainty was estimated using bootstrapping and presented using cost-effectiveness acceptability curves. Results Participants in the custom-made insole group experienced statistically significant more pain during activity at 26 weeks than participants in the usual care group (overall effect 1.06; $95 \% \mathrm{Cl} 0.36$ to 1.75). There were no significant differences between groups in other outcomes. Total societal costs in the custom-made insole group were non-significantly higher than in the usual care group (mean difference $€ 376 ; 95 \% \mathrm{Cl}-€ 1775$ to $€ 2038)$. The intervention with custom-made insoles was dominated by usual care by the GP (ie, more expensive and less effective) for pain during activity and quality of life outcomes. For the outcome pain at rest, the intervention was more expensive and more effective than usual care. However, the maximum probability of cost-effectiveness was only 0.59 at very high ceiling ratios.

Conclusions These findings show that that custom-made insoles are not cost-effective in comparison with GP-led usual care. Clinicians should be reserved in prescribing custom-made insoles for PHP as a primary intervention. Trial registration number NTR5346.

\section{INTRODUCTION}

Plantar heel pain (PHP), also known as plantar fasciopathy, is a common foot

\section{STRENGTHS AND LIMITATIONS OF THIS STUDY}

$\Rightarrow$ Study is based on a high-quality randomised controlled trial.

$\Rightarrow$ First study to perform this kind of analysis for treatment with custom-made insoles in patients with plantar heel pain.

$\Rightarrow$ Low rate of missing data for both outcome measures and costs.

$\Rightarrow$ Relatively short follow-up at 26 weeks.

$\Rightarrow$ Relatively low power to show significant differences in costs and effectiveness.

complaint. The incidence in Dutch general practice has been estimated to be 3.8 per 1000 person years. ${ }^{1}$ The clinical course of $\mathrm{PHP}$ is considered favourable with remission rates as high as $60 \%-80 \%$ at $12-24$ months after diagnosis. ${ }^{2}{ }^{3}$ Despite this favourable prognosis, the impact of PHP on the quality of life of patients is high, as the complaints have a large impact on every day activities. ${ }^{34}$ It is common for patients to try multiple treatments in primary care during the course of complaints. ${ }^{125}$ Commonly applied interventions in primary care include orthoses such as insoles. ${ }^{16}$ Orthoses, such as insoles, both prefabricated and custom-made are among the most commonly prescribed treatments to patients who present with PHP to their general practitioner (GP). ${ }^{1}$ However, this is a relatively expensive treatment option. ${ }^{7}$

The economic burden of PHP is estimated to be high. The annual healthcare costs related to PHP for third-party payers in the USA has been estimated to range from US $\$ 192$ to US $\$ 376$ million in 2007 (US $\$ 250$ US $\$ 488$ million in 2021). ${ }^{8}$ However, no data on lost productivity costs are available. One previous trial found that full length 
prefabricated orthoses were cost-effective compared with accommodative (half-length) orthoses. ${ }^{9}$ Another trial showed that prefabricated orthoses had a similar clinical effect as custom-made insoles at reduced costs. ${ }^{10}$ Considering the scarce resources available for healthcare it is important to establish whether monetary investments in treatment options for PHP are balanced by the additional health effects. Recently a randomised controlled trial to examine the cost-effectiveness of custom-made insoles by a podiatrist compared with usual care in patients with PHP was conducted. ${ }^{11}$ The effectiveness analysis showed that custom-made insoles resulted in a relevant increase in pain during activity compared with usual care by the GP. ${ }^{12}$ In this paper, the results of the cost-effectiveness analysis are presented.

\section{METHODS \\ Design}

A randomised controlled trial was conducted to estimate the cost-effectiveness of custom-made insoles compared with usual care. ${ }^{13}$ The design of the study will be summarised here; more details can be found elsewhere. ${ }^{1112}$

\section{Patient and public involvement}

No patients were involved in the planning and development of this study. The results will be disseminated directly to all participants via email.

\section{Study population}

Patients aged between 18 and 65 years who presented with PHP characterised as pain at the medial hind foot with a duration of at least 2 weeks to their GP were eligible for the trial. Exclusion criteria were recurrent PHP for more than 2 years, complaints caused by trauma, earlier treatment for PHP by a podiatrist or with insoles, suspected (by the GP or sports physician) (osteo)arthritis in the subtalar or talonavicular joint, suspected tarsal tunnel syndrome, suspected stress fractures, infections or tumours in the painful foot, presence of systemic diseases (such as ankylosing spondylitis, psoriasis or multiple sclerosis) and insufficient understanding of the Dutch language. After providing informed consent and completing the baseline questionnaire, participants were randomised by an independent person using a computer generated randomisation list (block randomisation with random block sizes between 3 and 10). Because we expected a larger difference between usual care and treatment with an insole, fewer patients were allocated to the usual care group.

\section{Treatment groups}

After inclusion, participants were randomised into three groups:

- GP-led usual (usual care).

- Referral to podiatrist for custom-made insole (custommade insole).

- Referral to podiatrist for sham insole (sham)
For the current cost-effectiveness analysis, we only analysed the participants in the usual care and custommade insole groups, because sham (placebo) is not considered a relevant alternative in cost-effectiveness studies. ${ }^{14}$

All participants received an information booklet on the use of pain medication and stretching and strength exercises for PHP. Participants allocated to the GP-led usual care group received usual care by their GP. This included watchful waiting, and any intervention the physician deemed necessary. GPs were encouraged not to refer their patients to a podiatrist during the course of the study.

Participants allocated to the custom-made insole group were referred to a podiatrist. Podiatrists were instructed to manufacture a custom-made insole as they would for any patient, but to withhold other interventions such as extracorporeal shockwave treatment. In total, three consultations with the podiatrist were offered to participants in the custom-made insole group. During the first consultation (within 1 week after randomisation), a standardised intake was performed by the podiatrist. The podiatrist was blinded to the participant's group allocation during this first consultation, but was informed about the allocation afterwards. Within 2 weeks after the intake, participants had a second consultation with the podiatrist to receive their custom-made insoles. A third check-up consultation was offered after at least 12 weeks after randomisation.

\section{Effectiveness outcomes}

All outcomes were reported by study participants in online questionnaires. The primary outcomes were pain at rest and pain during activity measured at baseline, and at 2, 4, 612 and 26 weeks of follow-up using a Numerical Rating Scale (NRS, 0-10). Because higher pain scores indicate worse outcomes, a negative difference in pain between groups indicates that the intervention is more effective than usual care. The 12-Item Short Form Health Survey (SF-12) and the EuroQol Five Dimension Scale Three levels (EQ-5D-3L) were measured at baseline, and at 12 and 26 weeks of follow-up. The SF-12 is a general quality of life measure that was converted to a Physical Component Score (PCS) and a Mental Component Score (MCS) ranging from 0 to $100 .^{15}$ Higher scores indicate better quality of life. The EQ-5D-3L is also a general quality of life measure. EQ-5D-3L health profiles were converted to utility values using the Dutch EQ-5D-3L tariff. ${ }^{16}$ Using the area under the curve method, quality-adjusted life-years (QALYs) were calculated by multiplying the amount of time a participant spent in a specific EQ-5D-3L health profile with the utility score associated with that health profile (QALY=timexutility). Changes between time points were linearly interpolated. Changes between time points were linearly interpolated.

\section{Cost outcomes}

Costs were measured from a societal perspective using online cost questionnaires with a 12 week recall period at baseline, and 12 and 26 weeks of follow-up. ${ }^{17}$ Costs 
included healthcare costs (ie, primary care, secondary care, medical devices and medication) and lost productivity costs (ie, absenteeism from paid and unpaid work, and presenteeism). Utilisation of healthcare services was valued using Dutch standard costs, if available. ${ }^{18}$ Otherwise, tariffs recommended by professional organisations were used. The costs of the insoles used in the study were reported by the podiatrists participating in the study. Medication was valued using the cost per daily defined dose. $^{19}$

Absenteeism from paid work was valued using the friction cost approach (FCA). The FCA assumes that sick employees are replaced after a certain period of timethe friction period-and that there are consequently no productivity losses anymore. A friction period of 12 weeks was used and absenteeism was valued using mean wage rates stratified by sex. ${ }^{18}$ Absenteeism from unpaid work was valued using a shadow price based on the costs for a legally employed cleaner. ${ }^{18}$ Presenteeism is defined as reduced efficiency due to health-related problems while being present at work. Efficiency while at work was rated using an NRS ranging from 0 (did my normal load of work) to 10 (did not do anything). Lost productivity due to presenteeism was then calculated by multiplying (1-efficiency score) with the number of hours that the participant was suffering from health-related problems. Lost productivity was subsequently valued using mean wage rates stratified by sex.

\section{Statistical analysis}

All analyses were performed according to the intentionto-treat principle. Missing cost and effectiveness data were imputed using multiple imputation by chained equations (MICE). An imputation model was created containing all variables included in the analysis models, and variables that were statistically different between groups at baseline, related to missingness or related to the outcomes. These variables were age, gender, body mass index, activity level according to SQUASH and bilateralism of pain. Note that the some variables used in the analysis models have not been included in the imputation model. Including a large number of variables leads to an overly complex imputation model that fails to converge, typically due to multicollinearity (ie, highly correlate covariates in the imputation model). To account for the skewed distribution of costs, predictive mean matching was used within the MICE procedure. The number of imputed datasets was increased until the fraction of missing information was less than $5 \%$, resulting in five imputed data sets. ${ }^{20}$ The complete imputed datasets were analysed separately as described below, after which results were pooled using Rubin's rules.

Differences in pain during rest and activity between groups over 26 weeks, and the SF-12 PCS and MCS scores were estimated using a longitudinal mixed model with two hierarchical levels (patients and time). Covariates were included if they changed the effect estimate by $>10 \%$. Differences in QALYs and total societal costs between groups were estimated using a linear regression model. Because costs are generally not normally distributed, statistical uncertainty surrounding costs and effectiveness was estimated using bias-corrected accelerated bootstrapping with 5000 replications. Incremental costeffectiveness ratios (ICERs) were calculated by dividing the difference in costs between the groups by the difference in effects. To ensure that a positive effect difference in the ICER calculation always indicates that the custommade insoles are more effective than usual care, the effect differences for the pain scores were multiplied by -1 . Uncertainty surrounding ICERs was shown by plotting the bootstrapped cost-effect pairs in cost-effectiveness planes. Cost-effectiveness acceptability curves were also estimated showing the probability that the custom-made insoles are cost-effective in comparison with GP-led usual care for different ceiling ratios. The ceiling ratio indicates the amount of money that society is willing to pay to gain one unit of effect. Sensitivity analyses included an economic evaluation from a healthcare perspective and an economic evaluation on a dataset of 103 participants ( $\mathrm{N}=65$ custom insole group, $\mathrm{N}=38$ usual care group) with complete data for all outcomes (ie, complete-case analysis).

All analyses were conducted using Stata/SE V.16.0.

\section{RESULTS}

In total, 185 patients were included in the trial after referral by their GP. Of the included patients, 46 were allocated to GP-led usual care, 70 to a custom-made insole (intervention) and 69 to sham insole (not analysed), leaving 116 patients for the cost-effectiveness analysis. Table 1 shows that there were no clinically relevant differences in baseline characteristics between the two analysed groups. In the custom-made insole group, three patients did not visit the podiatrist and four patients received their insole later than prescribed by the protocol. In the usual care group, four patients got custom-made insoles on their own initiative during the course of the study.

\section{Effectiveness outcomes}

Table 2 shows that participants in the custom-made insole group experienced significantly more pain during activity than participants in the usual care group over 26 weeks (overall effect $1.06 ; 95 \%$ CI 0.36 to 1.75 ). There were no statistically significant differences in the other effectiveness outcomes between the two groups (table 2).

\section{Cost outcomes}

Costs of visits to the GP and medication costs in the custom-made insole group were significantly lower statistically than in the usual care group (table 2). Participants in the custom-made insole group had statistically significant higher costs due to visits to the podiatrist and contacts with other healthcare providers than participants in the usual care group. The costs of the custom-made insoles were the largest contributor to total healthcare costs in 
Table 1 Baseline characteristics

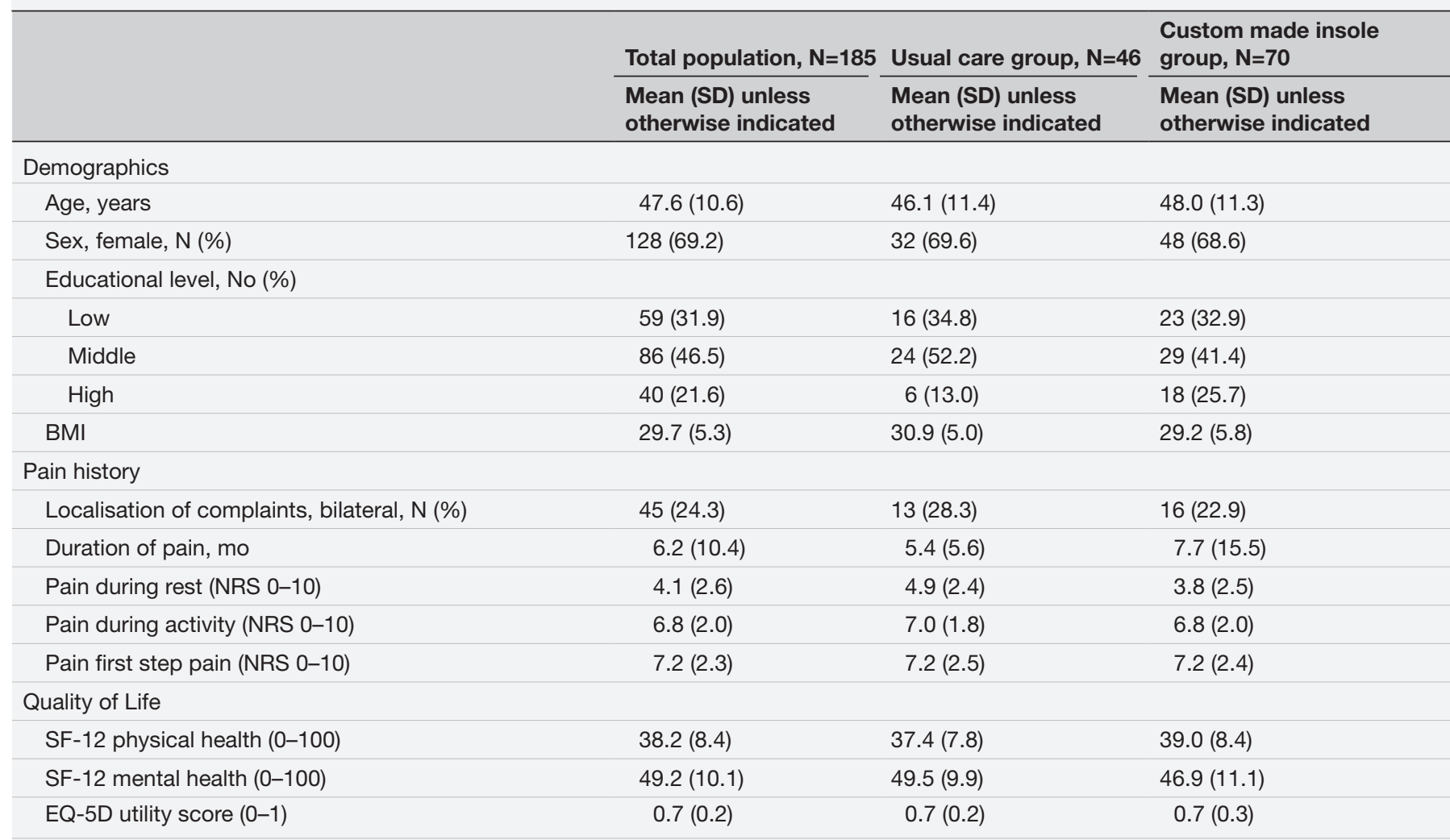

BMI, body mass index; EQ-5D, EuroQol Five Dimension Scale; mo, months; $n$, Number of participants; NRS, Numeric Rating Scale; SF-12, The 12Item Short Form Health Survey.

the custom-made insole group, and contributed most to the difference in healthcare costs between the groups (mean difference $€ 240 ; 95 \%$ CI $€ 159$ to $€ 427$ ). Medication costs in the custom-made insole group were lower than in the usual care group, even though the percentage of patients reporting the use of medication was similar in both groups. Participants in the custom-made insole group had lower costs related to absenteeism from paid and unpaid work than participants in the usual care group, but higher presenteeism costs. However, these differences were not statistically significant. Total societal costs after 26 weeks were $€ 2965$ in the custom-made insole group and $€ 2588$ in the usual care group, but this difference in total societal costs was not statistically significant (mean difference $€ 376$; $95 \%$ CI $-€ 1775$ to $€ 2038$ ).

\section{Cost-effectiveness outcomes}

Table 3 shows the outcomes of the cost-effectiveness analysis. For pain at rest, the ICER was -5661 indicating that on average $€ 5661$ needs to be invested in the custommade insole group to gain one point of improvement in pain at rest compared with the usual care group. The cost-effectiveness acceptability curve (figure 1) shows that the probability that the intervention is cost-effective in comparison with usual care is 0.35 at a ceiling ratio of $€ 0 /$ point of improvement in pain at rest and that this increases to a maximum probability of 0.50 at a ceiling ratio of $€ 6000 /$ point of improvement in pain at rest.
The maximum probability that the intervention is costeffective in comparison with usual care was 0.59 , but this requires very high ceiling ratios.

For pain during activity, the ICER was -356 indicating that on average $€ 356$ needs to be invested in the custommade insole group to lose one point of improvement in pain during activity compared with the usual care group. Thus, the intervention is dominated by usual care for pain during activity. The cost-effectiveness acceptability curve for QALY's (figure 2) shows that the probability that the intervention is cost-effective in comparison with usual care is 0.35 for all ceiling ratios.

For the SF-12 PCS, SF-12 MCS and QALYs, the results of the cost-effectiveness analysis were similar to the results for pain during activity, and indicated that the intervention was dominated by GP-led usual care. The cost-effectiveness acceptability curves (not shown) show that the probability that the intervention is cost-effective in comparison with GP-led usual care is 0.35 at a ceiling ratio of $€ 0 /$ additional unit of effect for all three quality of life measures. This probability decreases to 0.29 at a ceiling ratio of $€ 1000 /$ point improvement in SF-12 PCS, and 0.03 at a ceiling ratio of $€ 1000 /$ point improvement in SF-12 MCS. For QALYs, this probability remains around 0.35 for all possible ceiling ratios. The graphs for the cost-effectiveness planes for pain at rest and pain during activity can be viewed in online supplemental files 1 and 2). 
Table 2 Multiply imputed and adjusted outcomes after 26 weeks

\begin{tabular}{|c|c|c|c|}
\hline \multirow[b]{2}{*}{ Outcomes } & \multicolumn{2}{|l|}{ Mean (SE) } & \multirow[b]{2}{*}{ Mean difference $(95 \% \mathrm{CI})^{\star}$} \\
\hline & Custom-made insole $(n=70)$ & Usual care $(n=46)$ & \\
\hline \multicolumn{4}{|l|}{ Pain at rest (NRS $0-10) \dagger$} \\
\hline 2 weeks & $4.18(0.32)$ & $4.71(0.46)$ & \\
\hline 4 weeks & $4.00(0.32)$ & $3.92(0.47)$ & \\
\hline 26 weeks & $2.44(0.33)$ & $2.85(0.60)$ & \\
\hline \multicolumn{4}{|l|}{ Pain during activity (NRS $0-10) \S$} \\
\hline Baseline & $6.76(0.23)$ & $7.02(0.27)$ & $1.06(0.36$ to 1.75$) \ddagger$ \\
\hline 2 weeks & $6.53(0.24)$ & $5.68(0.49)$ & \\
\hline 26 weeks & $3.99(0.36)$ & $3.57(0.65)$ & \\
\hline \multicolumn{4}{|l|}{ SF-12 Physical component scoreף } \\
\hline Baseline & $39.00(1.01)$ & $37.36(1.15)$ & $-0.62(-3.47$ to 2.24$) \ddagger$ \\
\hline 12 weeks & $42.31(1.32)$ & $41.56(1.76)$ & \\
\hline 26 weeks & $43.63(1.34)$ & $45.43(2.18)$ & \\
\hline \multicolumn{4}{|l|}{ SF-12 Mental Component Score ${ }^{\star *}$} \\
\hline Baseline & $46.93(1.33)$ & $49.51(1.45)$ & $-2.79(-5.78$ to 0.20$) \ddagger$ \\
\hline 12 weeks & $47.00(1.37)$ & $47.83(1.87)$ & \\
\hline 26 weeks & $47.34(1.30)$ & $48.92(1.45)$ & \\
\hline Quality-adjusted life-year (0-1) & $0.35(0.01)$ & $0.35(0.01)$ & $-0.00(-0.04$ to 0.03$)$ \\
\hline Secondary care & $8(3)$ & $8(4)$ & $0(-10$ to 9$)$ \\
\hline Medication & $6(2)$ & $36(11)$ & $-30(-56$ to -15$)$ \\
\hline Intervention costs & $205(11)$ & $0(0)$ & 205 (180 to 224$)$ \\
\hline Total healthcare costs & $375(55)$ & $135(32)$ & 240 (159 to 427$)$ \\
\hline \multicolumn{4}{|l|}{ Non-healthcare costs $(€)$} \\
\hline \multicolumn{4}{|l|}{ Lost productivity } \\
\hline Absenteeism & $232(137)$ & $653(475)$ & $-421(-2117$ to 135$)$ \\
\hline Presenteeism & $2085(455)$ & $1351(595)$ & 734 (-524 to 1953$)$ \\
\hline Unpaid work & $273(121)$ & 449 (183) & $-176(-577$ to 160$)$ \\
\hline Total non-healthcare costs & $2590(517)$ & $2453(905)$ & 137 (-2490 to 1656$)$ \\
\hline Total societal costs $(€)$ & $2965(520)$ & 2588 (909) & 376 (-1775 to 2038$)$ \\
\hline
\end{tabular}

*Uncertainty around cost differences estimated using the non-parametric bootstrap.

†Analysis is also adjusted for educational level, bilateralism of pain, other musculoskeletal pain, self-reported illness in last 12 months, physical component of the SF-12 at baseline, mental component of the SF-12 at baseline, pain score during activity and the disability subscore of the FFI at baseline.

fOverall effect over time.

§Analysis is also adjusted for duration of pain, bilateralism of pain, the physical component of the SF-12 at baseline, pain score during activity at baseline, selfreported illness in last 12 months and other musculoskeletal pain.

ๆAnalysis is also adjusted for educational level, bilateralism of pain, pain score at rest at baseline, pain score during activity at baseline, self-reported illness in last 12 months and the disability subscore of the FFI at baseline.

${ }^{* *}$ Analysis is also adjusted for duration of pain, bilateralism of pain, the physical component of the SF-12 at baseline, pain score during activity at baseline, selfreported illness in last 12 months and other musculoskeletal pain.

FFI, Foot Function Index; NRS, Numeric Rating Scale; SF-12, The 12-Item Short Form Health Survey. 
Table 3 Outcomes for the cost-effectiveness analysis

\begin{tabular}{|c|c|c|c|c|c|c|c|}
\hline \multirow[b]{2}{*}{ Outcome } & \multirow[b]{2}{*}{$\Delta \mathrm{C}(95 \% \mathrm{Cl})^{*}$} & \multirow[b]{2}{*}{$\Delta \mathrm{E}(95 \% \mathrm{Cl}) \dagger$} & \multirow[b]{2}{*}{ ICER‡ } & \multicolumn{4}{|c|}{ CE plane } \\
\hline & & & & NE & SE & sw & NW \\
\hline \multicolumn{8}{|l|}{ Main analysis: societal perspective } \\
\hline Pain during activity§ & $376(-1775$ to 2038$)$ & $-1.06(-1.69$ to -0.43$)$ & -356 & $0 \%$ & $0 \%$ & $35 \%$ & $65 \%$ \\
\hline Physical Component Score (PCS) & 376 (-1775 to 2038$)$ & $-0.62(-3.22$ to 1.98$)$ & -611 & $18 \%$ & $12 \%$ & $22 \%$ & $48 \%$ \\
\hline QALYs (0-1) & $376(-1775$ to 2038$)$ & $-0.0016(-0.027$ to 0.024$)$ & -236420 & $27 \%$ & $17 \%$ & $17 \%$ & $38 \%$ \\
\hline \multicolumn{8}{|l|}{ SA1: Healthcare perspective } \\
\hline Pain at res§ & 240 (146 to 389$)$ & $-0.066(-0.58$ to 0.72$)$ & -3605 & $59 \%$ & $0 \%$ & $0 \%$ & $41 \%$ \\
\hline Pain during activity§ & 240 (146 to 389$)$ & $-1.06(-1.69$ to -0.43$)$ & -226 & $0 \%$ & $0 \%$ & $0 \%$ & $100 \%$ \\
\hline PCS & 240 (146 to 389$)$ & $-0.62(-3.22$ to 1.98$)$ & -389 & $30 \%$ & $0 \%$ & $0 \%$ & $70 \%$ \\
\hline Pain at rest§ & 834 (-1796 to 2561$)$ & $-0.0074(-0.71$ to 0.68$)$ & -112053 & $70 \%$ & $6 \%$ & $5 \%$ & $19 \%$ \\
\hline Pain during activity§ & $834(-1796$ to 2561$)$ & $-1.16(-1.80$ to -0.54$)$ & -720 & $50 \%$ & $0 \%$ & $10 \%$ & $40 \%$ \\
\hline PCS & $834(-1796$ to 2561$)$ & $-0.95(-3.18$ to 1.42$)$ & -878 & $31 \%$ & $10 \%$ & $19 \%$ & $40 \%$ \\
\hline MCS & $834(-1796$ to 2561$)$ & $-3.36(-6.33$ to -0.63$)$ & -248 & $50 \%$ & $1 \%$ & $10 \%$ & $39 \%$ \\
\hline QALYs (0-1) & $834(-1796$ to 2561$)$ & $-0.0013(-0.027$ to 0.026$)$ & -658249 & $68 \%$ & $5 \%$ & $5 \%$ & $22 \%$ \\
\hline
\end{tabular}

*Uncertainty around cost differences estimated using the non-parametric bootstrap.

†Overall effect over time.

$\ddagger$ The ICER presented was computed using the unrounded values for cost and effect. In the table, rounded values are presented.

$\S$ To ensure that a positive effect difference in the ICER calculation always indicates that the custom-made insoles are more effective than usual care, the effect differences for the pain scores were multiplied by -1 .

IA dataset of 103 participants with complete data on all relevant outcomes was used.

$\triangle \mathrm{C}$, difference in costs between the two groups; CE-plane, cost-effectiveness plane; $\triangle \mathrm{E}$, difference in effectiveness outcome between the two groups; ICER, incremental cos-effectiveness ratio; NE, North-East Quadrant; NW, North-West Quadrant; QALY, quality-adjusted life-year; SA, sensitivity analysis; SE, South-East Quadrant; SF-12, The 12-Item Short Form Health Survey; SW, South-West Quadrant.

\section{Sensitivity analyses}

From the healthcare perspective, costs in the custommade sole group were on average $€ 240$ higher than in the usual care group. Due to less statistical uncertainty in healthcare costs, this difference was statistically significant. As a consequence, the probability that the intervention is dominated by control increased compared with the main analysis to $41 \%$ for pain at rest, $55 \%$ for QALYs, $70 \%$ for the SF-12-PCS, 98\% for the SF-12-MCS and 100\% for pain during activity.

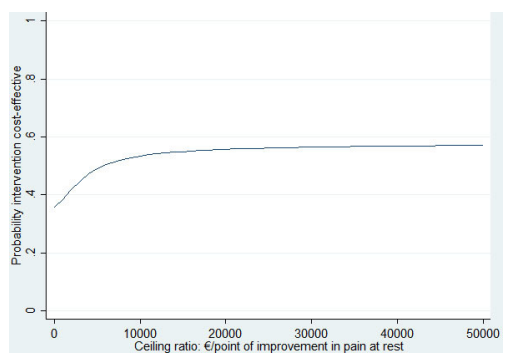

Figure 1 The cost-effectiveness acceptability curve for pain at rest.
In the complete-case analysis, costs in the custom-made sole group were on average $€ 834$ higher than in the usual care group, although this difference was not statistically significant. The differences in effectiveness between the

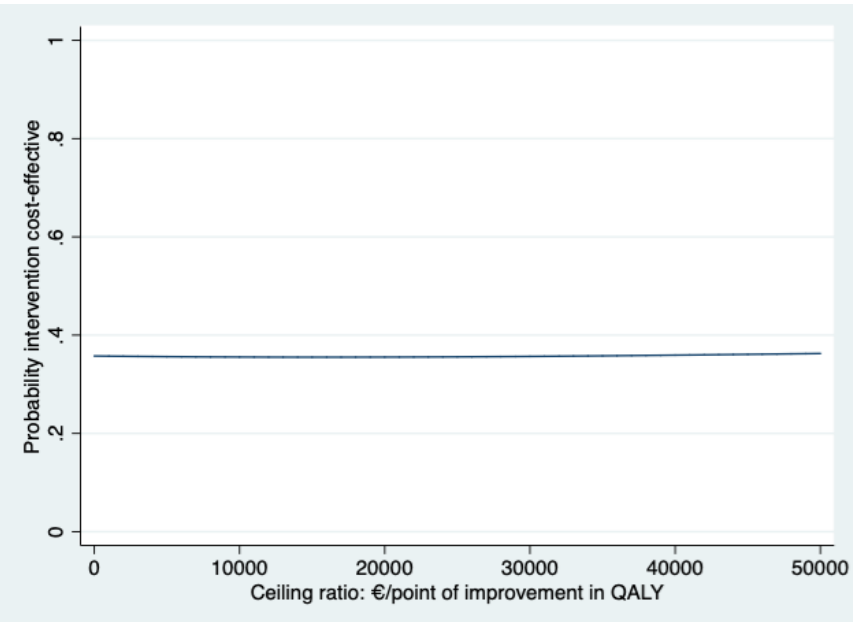

Figure 2 The cost-effectiveness acceptability curve for quality-adjusted life-years (QALY). 
groups increased slightly for all effectiveness measures except QALYs. The probability that the custom-made soles are cost-effective in comparison with GP-led usual care ranges from $10 \%$ (QALYs) to $29 \%$ for SF-12-PCS at a ceiling ratio of $€ 0 /$ additional unit of effect.

\section{DISCUSSION}

\section{Principal findings}

Our findings show that custom-made insoles are not cost-effective in comparison to GP-led usual care. In the custom-made insole group, podiatrist costs were higher than in the usual care group, whereas GP and physiotherapy costs were higher in the usual care group. Costs of secondary care (such as radiology or referrals) were similar in the two groups. However, medication costs in the custom-made insole group were lower than in the usual care group, although the percentage of patients reporting the use of medication was similar in both groups. The cost-effectiveness analyses showed that treatment with custom-made insoles was dominated by GP-led usual care (ie, more expensive and less effective) for pain during activity and quality of life outcomes. For the outcome pain at rest, treatment with custom-made insoles was also more expensive, but more effective than GP-led usual care. However, the maximum probability of cost-effectiveness was only 0.59 at very high ceiling ratios. The greatest contributor to healthcare costs were the costs of the custom-made insoles and the podiatric treatment. This is in line with previous literature where costs for insoles were also relatively high. ${ }^{810}$ This was despite the fact that costs specifically related to the conduct of the current study (eg, the costs of producing threedimensional imprints of the foot) were eliminated from the analysis. Thus, only costs for the production of the insole and for the consultations were included.

\section{Comparison to existing literature}

To our knowledge, there are no other studies that performed a cost-effectiveness analysis on custom-made insoles in patients with PHP. In the study of Ring and Otter from 2014, the costs of custom-made orthoses were compared with prefabricated orthoses. ${ }^{10}$ Although they concluded that the prefabricated orthoses gave similar results at reduced cost, they did not perform an incremental cost-effectiveness analysis (ie, they only analysed the effectiveness and cost outcomes separately) and did not include lost productivity costs in their analysis. Therefore, our cost-effectiveness analysis adds valuably knowledge to the existing evidence base on custom-made insoles in the treatment of PHP.

\section{Strengths and limitations}

The main strength of our study is that it is the first to provide evidence on the cost-effectiveness of custommade insoles compared with usual GP care in patients with PHP from a societal perspective alongside a highquality randomised controlled trial. Another strength is that the study had a pragmatic design that mimicked actual clinical practice as closely as possible. This greatly improves the generalisability of the findings greatly. A final strength is the low rate of missing data for both effectiveness and costs. To avoid bias due to selective dropout, multiple imputation was used to impute missing observations. Multiple imputation is generally considered the most valid method to impute missing data. ${ }^{21}$

A limitation of the study may be that the quality of life instruments used are not sensitive to changes in the severity of PHP complaints. However, considering the great impact such complaints have on the daily life of patients, we think that this not likely. A second limitation is that costs and effects were measured over a period of 26 weeks. This may not be enough to observe all potential benefits and costs related to the treatments, since studies show that it may take up to $12-16$ months to recover from PHP. ${ }^{23}$ Finally, the power of the study was too low to show statistically significant differences in costs and effects, which is often the case. ${ }^{22}$ Also the small sample size of the study has led to imputation of data being necessary. Therefore, it is important to focus more on the size of the cost differences and the uncertainty surrounding these differences. In the current study, cost differences in lost productivity costs and total societal costs were relatively large, but there was also much uncertainty surrounding these differences. Another limitation is the fact that the participants, who were allocated to the custommade insole, were blinded to the type of insole they received (sham vs custom-made). This could have affected the trust they had in the treatment and the way they reported their outcomes. Therefore, the comparison made between the custom-made insole and GP-led usual care may differ from clinical practice. Also, the fact that this study was performed within the Dutch healthcare system may affect the generalisability. Differences in healthcare systems internationally can translate to differences in costs for similar interventions. For example, all Dutch citizens are obliged to have a GP who acts as a gatekeeper for more specialised forms of healthcare. We feel that our conclusions need to be seen in light of these differences, however, since custom insoles are relatively expensive in all countries, the comparison to "usual care', which contains more of an wait-and-see approach and choice from a variety of cheaper interventions, holds up in other healthcare systems as well. Regardless of which healthcare provider is in a position to provide the 'usual care approach'.

\section{Implications for clinical practice}

Based on our study, we conclude that custom-made insoles are not cost-effective in comparison with GP-led usual care. We think that this should be taken into account when making clinical decisions as the findings of this study indicate that clinicians should be reserved in prescribing custom-made insoles to patients with PHP as a primary intervention.

Acknowledgements We thank the participants who volunteered to participate in the study and the GP's that recruited participants in their practices. Also we thank 
the podiatrists who were involved in the consensus meeting on the interventions and who provided the intervention to the participants.

Contributors NR contributed to the study design, recruitment of participants, management of study proceedings, data collection and drafting and revision of the manuscript. MvM, SMAB-Z, PB, MEA and JB contributed to the study conception and design, and drafting and revision of the manuscript. NR and JB are responsible for the overall content as the guarantor. The corresponding author attests that all listed authors meet authorship criteria and that no others meeting the criteria have been omitted.

Funding This trial is funded by The Netherlands Organisation for Health Research and Development (ZonMW) under number: 839110 008. The Dutch Association of Podiatrist (NVvP) funded the intervention provided to patients in the insole and the sham group.

Competing interests All authors have completed the ICMJE uniform disclosure form at www.icmje.org/coi_disclosure.pdf and declare: support from ZonMW and the NVvP for the submitted work. SMAB-Z declares: grants from The Netherlands Organisation for Health Research and Development (ZonMW), other from The Dutch Association of Podiatrists (NVvP), during the conduct of the study; grants from The Netherlands Organisation for Health Research and Development, CZ, European Union, Foreum, Dutch Arthritis Association, personal fees from 0steoarthritis Research Society International (OARSI), personal fees from Pfizer, outside the submitted work

Patient consent for publication Not applicable.

Ethics approval The Medical Ethics Committee (number 2015-253) of the Erasmus Medical Centre in Rotterdam has approved this study. This permission includes the multicentre nature of this study, that is, approval was given to recruit patients at all sites.

Provenance and peer review Not commissioned; externally peer reviewed.

Data availability statement Data are available on reasonable request. Data and statistical analysis plan are available through mediated access by contacting Marienke van Middelkoop: m.vanmiddelkoop@erasmusmc.nl.

Supplemental material This content has been supplied by the author(s). It has not been vetted by BMJ Publishing Group Limited (BMJ) and may not have been peer-reviewed. Any opinions or recommendations discussed are solely those of the author(s) and are not endorsed by BMJ. BMJ disclaims all liability and responsibility arising from any reliance placed on the content. Where the content includes any translated material, BMJ does not warrant the accuracy and reliability of the translations (including but not limited to local regulations, clinical guidelines, terminology, drug names and drug dosages), and is not responsible for any error and/or omissions arising from translation and adaptation or otherwise.

Open access This is an open access article distributed in accordance with the Creative Commons Attribution Non Commercial (CC BY-NC 4.0) license, which permits others to distribute, remix, adapt, build upon this work non-commercially, and license their derivative works on different terms, provided the original work is properly cited, appropriate credit is given, any changes made indicated, and the use is non-commercial. See: http://creativecommons.org/licenses/by-nc/4.0/.

ORCID iD

Nadine Rasenberg http://orcid.org/0000-0001-7190-1779

\section{REFERENCES}

1 Rasenberg N, Bierma-Zeinstra SM, Bindels PJ, et al. Incidence, prevalence, and management of plantar heel pain: a retrospective cohort study in Dutch primary care. Br J Gen Pract 2019;69:e801-8.
2 Buchbinder R. Clinical practice. Plantar fasciitis. N Engl J Med 2004:350:2159-66.

3 Digiovanni BF, Nawoczenski DA, Malay DP, et al. Plantar fasciaspecific stretching exercise improves outcomes in patients with chronic plantar fasciitis. A prospective clinical trial with two-year follow-up. J Bone Joint Surg Am 2006;88:1775-81.

4 Irving DB, Cook JL, Young MA, et al. Impact of chronic plantar heel pain on health-related quality of life. J Am Podiatr Med Assoc 2008;98:283-9.

5 Riddle DL, Schappert SM. Volume of ambulatory care visits and patterns of care for patients diagnosed with plantar fasciitis: a national study of medical doctors. Foot Ankle Int 2004;25:303-10.

6 Nahin RL. Prevalence and pharmaceutical treatment of plantar fasciitis in United States adults. J Pain 2018;19:885-96.

7 Tran K, Spry C. Custom-Made foot Orthoses versus prefabricated foot Orthoses: a review of clinical effectiveness and costeffectiveness. Canadian Agency for Drugs and Technologies in Health, 2019.

8 Tong KB, Furia J. Economic burden of plantar fasciitis treatment in the United States. Am J Orthop 2010;39:227-31.

9 Rome K, Gray J, Stewart F, et al. Evaluating the clinical effectiveness and cost-effectiveness of foot orthoses in the treatment of plantar heel pain: a feasibility study. J Am Podiatr Med Assoc 2004:94:229-38.

10 Ring K, Otter S. Clinical efficacy and cost-effectiveness of bespoke and prefabricated foot orthoses for plantar heel pain: a prospective cohort study. Musculoskeletal Care 2014;12:1-10.

11 Rasenberg N, Fuit L, Poppe E, et al. The STAP-study: the (cost) effectiveness of custom made orthotic insoles in the treatment for plantar fasciopathy in general practice and sports medicine: design of a randomized controlled trial. BMC Musculoskelet Disord 2016;17:31

12 Rasenberg N, Bierma-Zeinstra SMA, Fuit L, et al. Custom insoles versus sham and GP-led usual care in patients with plantar heel pain: results of the STAP-study - a randomised controlled trial. Br J Sports Med 2021;55:272-8.

13 World Medical Association. World Medical association Declaration of Helsinki: ethical principles for medical research involving human subjects. JAMA 2013;310:2191-4.

14 Jönsson L, Sandin R, Ekman M, et al. Analyzing overall survival in randomized controlled trials with crossover and implications for economic evaluation. Value Health 2014;17:707-13.

15 Ware J, Kosinski M, Keller SD. A 12-Item short-form health survey: construction of scales and preliminary tests of reliability and validity. Med Care 1996;34:220-33.

16 Lamers LM, Stalmeier PFM, McDonnell J, et al. [Measuring the quality of life in economic evaluations: the Dutch EQ-5D tariff]. Ned Tijdschr Geneeskd 2005:149:1574-8.

17 Bouwmans C, Krol M, Severens H, et al. The iMTA productivity cost questionnaire: a standardized instrument for measuring and Valuing health-related productivity losses. Value Health 2015;18:753-8.

18 Kanters TA, Bouwmans CAM, van der Linden N, et al. Update of the Dutch manual for costing studies in health care. PLoS One 2017;12:e0187477.

19 Zorglnstituut Nederland. Medicijnkosten, 2019. Available: www. medicijnkosten.nl

20 White IR, Royston P, Wood AM. Multiple imputation using chained equations: issues and guidance for practice. Stat Med 2011;30:377-99

21 Burton A, Billingham LJ, Bryan S. Cost-effectiveness in clinical trials: using multiple imputation to deal with incomplete cost data. Clin Trials 2007;4:154-61.

22 Briggs A. Economic evaluation and clinical trials: size matters. $B M J$ 2000;321:1362-3. 